\title{
Differential Regulation of Primitive Myelopoiesis in the Zebrafish by Spi-1/Pu.1 and C/ebp1
}

\author{
Fengyun Su, ${ }^{1}$ Marianne A. Juarez, ${ }^{1}$ Christopher L. Cooke, ${ }^{1}$ Lisa LaPointe, ${ }^{1}$ Jordan A. Shavit, ${ }^{2}$ \\ Jennifer S. Yamaoka, ${ }^{2}$ and Susan E. Lyons ${ }^{1}$
}

\begin{abstract}
The zebrafish has become a powerful tool for analysis of vertebrate hematopoiesis. Zebrafish, unlike mammals, have a robust primitive myeloid pathway that generates both granulocytes and macrophages. It is not clear how this unique primitive myeloid pathway relates to mammalian definitive hematopoiesis. In this study, we show that the two myeloid subsets can be distinguished using RNA in situ hybridization. Using a morpholino-antisense gene knockdown approach, we have characterized the hematopoietic defects resulting from knockdown of the myeloid transcription factor gene $p u .1$ and the unique zebrafish gene c/ebp1. Severe reduction of pu.1 resulted in complete loss of primitive macrophage development, with effects on granulocyte development only with maximal knockdown. Reduction of $c / e b p 1$ did not ablate initial macrophage or granulocyte development, but resulted in loss of expression of the secondary granule gene lys $C$. These data reveal strong functional conservation of pu.1 between zebrafish primitive myelopoiesis and mammalian definitive myelopoiesis. Further, these results are consistent with a conserved role between $c / e b p 1$ and mammalian C/EBPE, whose ortholog in zebrafish has not been identified. These studies validate the examination of zebrafish primitive myeloid development as a model for human myelopoiesis, and form a framework for identification and analysis of myeloid mutants.
\end{abstract}

\section{Introduction}

$\mathbf{I}$ N MAMMALS, MYELOPOIESIS OCCURS during definitive hematopoiesis, through progressive commitment and differentiation steps from pluripotent hematopoietic stem cells to common myeloid progenitors that ultimately generate terminally differentiated erythroid, granulocyte, and macrophage/monocyte lineages. The genes that direct this process, including scl, spi-1/pu.1 (hereafter called pu.1), c/ebps, and gata1, have been shown to play important roles not only in normal hematopoiesis but also in leukemogenesis. $^{1-5}$

Zebrafish have become a powerful vertebrate model for analyses of hematopoiesis. Due to the easy visibility of erythroid cells, over 26 hematopoietic mutants with abnormalities in erythroid populations have been identified. ${ }^{6}$ The cloning of the genes mutated in these defects has identified both known and novel genes required for stem cell development, and development and/or maintenance of the erythroid lineage. ${ }^{7,8}$ In contrast to the early hematopoietic screens that successfully identified alterations in erythropoiesis, mutagenesis screens to analyze myeloid deficiencies are in the early stages. The ability to design directed screens for myeloid defects requires a better understanding of normal myelopoiesis in the zebrafish.

Zebrafish develop both macrophages and granulocytes during primitive hematopoiesis, ${ }^{9-11}$

\footnotetext{
${ }^{1}$ Division of Hematology-Oncology and Cellular and Molecular Biology Program, Department of Internal Medicine, University of Michigan, Ann Arbor, Michigan.

${ }^{2}$ HHMI and Life Sciences Institute, University of Michigan, Ann Arbor, Michigan.
} 
while mammals generate largely erythroid cells, with a small number of primitive macrophages and no granulocytes, during primitive hematopoiesis. ${ }^{9-12}$ Due to the ease of examining early events in the zebrafish, primitive myelopoiesis is being used in most screens to assess myelopoiesis, although the parallels that can be drawn between primitive myelopoiesis in zebrafish and definitive myelopoiesis in mammals are not known.

In the zebrafish, early markers of hematopoiesis are first evident by the 1-3 somite stage or 11 hours postfertilization (hpf) in two regions, the anterior lateral mesoderm (ALM) region near the head and the posterior lateral mesoderm (PLM), which later forms the intermediate cell mass (ICM). ${ }^{13}$ Erythroid development takes place in the PLM/ICM, while macrophages develop from the ALM ${ }^{10}$ and granulocytes may develop from the ALM and PLM. ${ }^{9,11}$

Transcription factors essential for myeloid development in mammals include PU.1 and a number of C/EBP family members. PU.1 is an Ets-family transcription factor known to be essential for macrophage and B lymphoid development and some aspects of granulocyte differentiation in mammals. ${ }^{14,15}$ The C/EBP family of transcription factors includes several members involved in hematopoietic development. Orthologs of C/ebpa and C/ebpb have been identified in the zebrafish. ${ }^{16}$ Interestingly, an ortholog for $\mathrm{C} / \mathrm{ebpe}$, required for terminal maturation of granulocytes and expression of secondary granule proteins in mammals, has not been identified in the zebrafish. However, another C/ebp family member, C/ebp1, was identified in zebrafish and is expressed specifically in myeloid cells. ${ }^{17}$ The functional role of $\mathrm{C} / \mathrm{ebp} 1$ in myeloid development has not yet been explored. Here we characterize the myeloid subsets present during primitive hematopoiesis and examine the functional roles of Pu.1 and C/ebp1 through a morpholino (MO)based knockdown approach.

\section{Results}

Two myeloid subsets can be distinguished in primitive zebrafish hematopoiesis

To characterize the myeloid cell populations present in zebrafish embryos, whole-mount embryos were examined by double RNA in situ hybridizations using the myeloid-specific markers l-plastin $(l c p / l-p l),{ }^{10}$ myeloperoxidase (mpo), ${ }^{9}$ lysozyme C (lys C), ${ }^{18}$ macrophage colonystimulating factor receptor (csf1r/c-fms), ${ }^{19,20}$ and CCAAT/enhancer binding protein 1 (c/ebp1). ${ }^{17}$

Previous work correlated the appearance of $l-p l$ expression with regions in which macrophages were visualized in live embryos. ${ }^{10,20}$ Further, $l-p l$ has been shown to be coexpressed in at least a subset of cells expressing $c / e b p 1^{17}$ and lys $C_{,}^{18}$ while the majority of mpo-expressing cells were found to be distinct from $l-p l$. However, further studies to distinguish clearly amongst these multiple subsets have not been performed.

Beginning at approximately $22-24 \mathrm{hpf}$, two populations of cells could be distinguished by staining with $l-p l, c$-fms, mpo, and lys C. One population present in both the tail and yolk sac stained with mpo and lys $C$ (Fig. 1C) and did not express l-pl (Fig. 1A, B) or c-fms (Fig. 1D, E). The second population, also present in yolk sac and tail, costained with both $l-p l$ and $c$-fms (Fig. 1F), but did not stain with lys $C$ or mpo (Fig. 1D, E). We conclude that the cells costaining with $l-p l$ and $c-f m s$ are likely macrophages given that $c$-fms is expressed specifically in macrophages in mammals ${ }^{21}$ and characterization of macrophages in zebrafish has correlated the movement of these macrophages on video microscopy with the positions of $l-p l(+)$ cells by RNA in situ hybridization. ${ }^{10}$

mpo expression has been demonstrated in adult zebrafish granulocytes by in situ hybridizations in kidney sections. ${ }^{9}$ Thus, the mpo $(+) /$ lys $C(+)$ population likely marks granulocytes in embryos. While mpo and lys $C$ staining was largely coincident, prior to circulation (between 21 somites and $24 \mathrm{hpf}$ ) there was a population of mpo-staining cells that did not express lys $C$. Single mpo-expressing cells were in the ICM only (Fig. 2A), while double-staining mpo $(+) /$ lys $C(+)$ cells were only present over the yolk sac (Fig. 2B). The population of mpo $(+) /$ lys $C$ $(-)$ cells in the ICM did not costain with gata1 (Fig. 2C) and was therefore clearly distinct from erythroid progenitors. In mammals, myeloperoxidase is a primary granule protein, while lysozyme is found in both primary and secondary granules. We therefore postulate that 

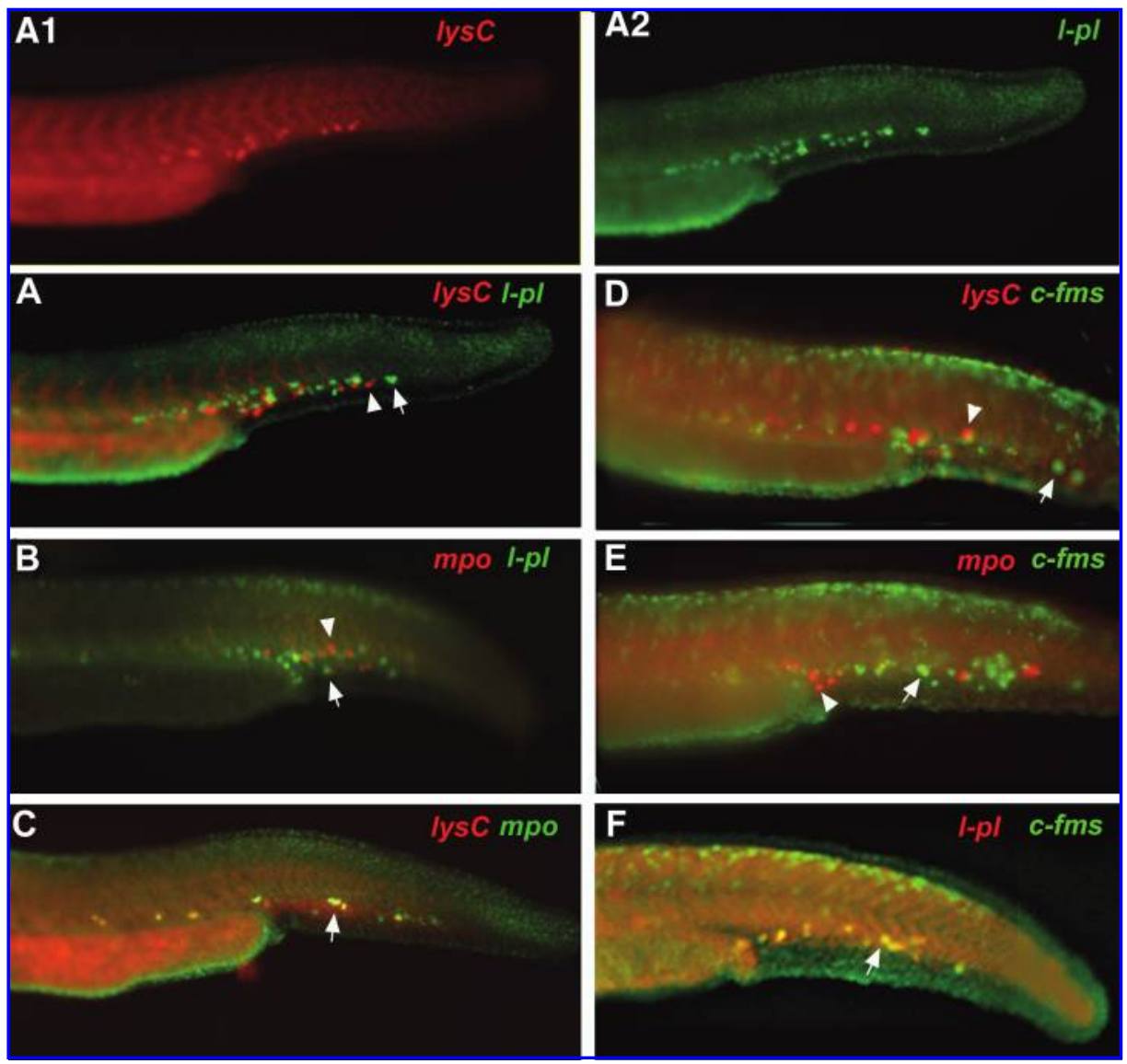

FIG. 1. Double in situ hybridization with myeloid markers. (A-F) $28 \mathrm{hpf}$ embryos, lateral views of the tail, with embryos oriented with the head to the left. Double in situ hybridizations were performed with dual fluorescent markers, as indicated. Photographs taken with each filter were overlaid using Adobe Photoshop and are shown in (A-F). In (A1) and (A2), individual pictures of an in situ hybridized embryo show signal from lys $C$ taken using the rhodamine filter (A1) or signal from $l-p l$ using the FITC filter (A2). There is no "bleed-through" of signal seen with each filter. (A) is the merged photo using (A1) and (A2). (A), (B), (D), and (E) signals are completely nonoverlapping (white arrows and arrowheads), while in (C) and (F), overlapping signals result in yellow color (white arrows).

the single mpo-staining population may represent an earlier step in granulocyte maturation prior to lys $C$ expression.

In a previous work we identified a $\mathrm{C} / \mathrm{ebp}$ family member expressed only in myeloid cells, which we called $c / e b p 1 .{ }^{17} c / e b p 1$ does not have an ortholog in mammals, but has an identified ortholog in at least one other fish species (GenBank accession no. AB049814). We have shown previously that a subset of cells expressing $l-p l$ coexpress $c / e b p 1{ }^{17}$ To determine whether the mpo/lys $C$ doubly positive population expressed $c / e b p 1$, two-color staining in situ hybridizations were performed at $28 \mathrm{hpf}$ with $c / e b p 1$ and lys C. Two-color staining demonstrated coexpression of $c / e b p 1$ with a subset of lys C-expressing cells in the tail (Fig. 2D) and yolk sac (data not shown). Thus, expression of $c / e b p 1$ occurs in a subset of the cells of both the macrophage and granulocyte lineages.

\section{Macrophage development and granulocyte} development are differentially affected

in pu. 1 MO-injected embryos

To examine the requirement for Pu.1 in zebrafish primitive myeloid development, pu.1 was targeted in zebrafish embryos using MOs recognizing the pu.1 translation initiation site ( $p u .1$ init) or the exon 5 splice donor site ( $p u .1$ ex 5), which is the most proximal exon to the Ets domain, required for Pu.1 function.

To examine the efficacy of the pu.1 ex $5 \mathrm{MO}$, we analyzed splice products from pu.1 ex 5 


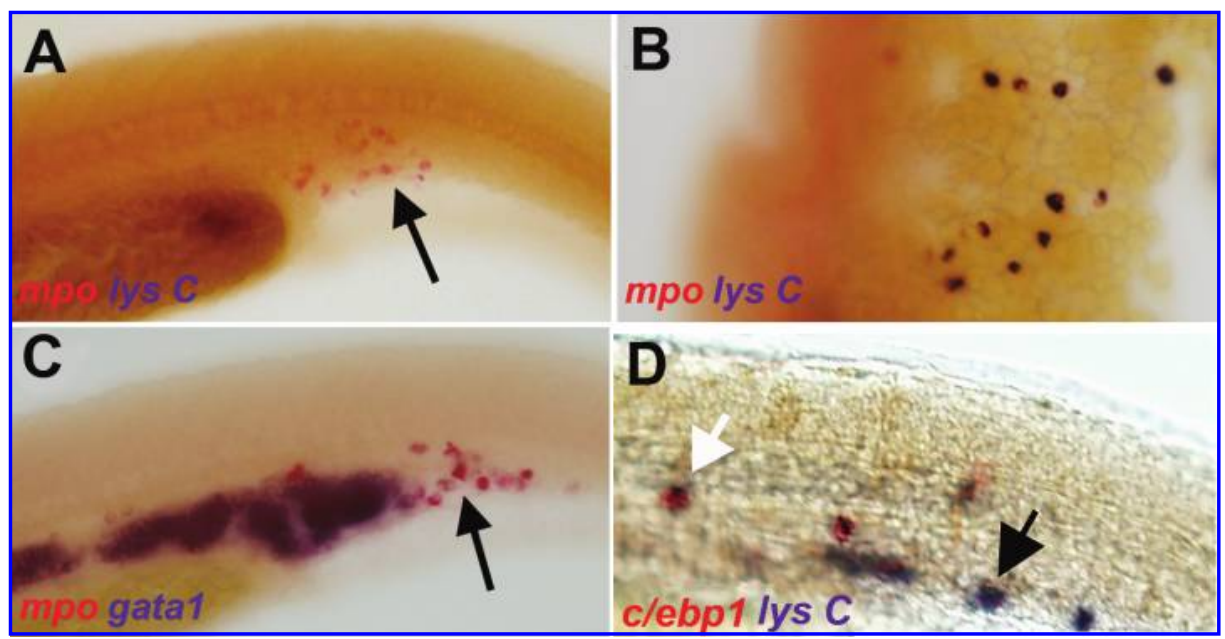

FIG. 2. Double in situ hybridization with myeloid markers and erythroid marker. (A-D) Double in situ hybridizations with fast red colorimetric reaction and BM-Purple (Roche) color reaction with probes as marked. Twenty-twohpf embryos are shown in (A-C) in lateral views, and a $28 \mathrm{hpf}$ embryo is shown in (D) in lateral view. (A) ICM of embryo double stained with mpo (red) and lys $C$ (purple). (B) Yolk sac of embryo shown in (A) shows lys $C$ staining. mpo staining is coincident with lys $C$, with no cells staining with red only. (C) Tail of embryo double stained with mpo (red) and gata1 (purple). Cells staining only with mpo are shown by the black arrow. The mpo-staining cells are distinct from the gata1 (+) erythroid population. (D) Tail of embryo stained with $c / e b p 1$ (red) and lys $C$ (purple). Cells expressing both $c / e b p 1$ and lys $C$ are marked with a white arrow, while cells expressing only lys $C$ are marked with a black arrow.

MO-injected embryos. RNA at 21 somites was harvested from pu.1 ex $5 \mathrm{MO}$-injected and pu.1 ex 5-misinjected embryos. Polymerase chain reaction (PCR) primers spanning the exon 5 splice donor site were used to amplify pu.1 splice products (Fig. 3A). Three products were detected from RNA recovered from pu.1 ex 5 $\mathrm{MO}-$ injected samples, and sequencing revealed that each product was distinct from the wild-type product amplified from pu.1 ex 5misinjected or uninjected embryos (Fig. 3A, B). There were three splice variants utilizing different cryptic splice donors and either the exon 6 splice acceptor or a cryptic acceptor (Fig. 3B, C). Each of the products resulted in a predicted translation product lacking the highly conserved Ets-DNA binding domain (Fig. 3C). Thus, all products would be expected to result in nonfunctional, prematurely truncated proteins. There was no wild-type product detected.

Injection of pu.1 init (data not shown) or pu.1 ex 5 (Fig. 4) resulted in complete loss of $l-p l$ in $86 \%$ of embryos $(n=82)$ compared to animals injected with a control, mismatch $\mathrm{MO}$, in which $100 \%$ of embryos expressed $l-p l(p<0.001)$ (Fig. $4 \mathrm{~A}, \mathrm{~B})$. There was also loss of myeloid c-fms staining in $100 \%$ of injected embryos $(n=38)$ (Fig. 4D) compared to controls, which all stained over the yolk sac for $c$-fms $(n=30 ; p<0.001)$ (Fig. 4C). Expression of $c$-fms in previously described nonhematopoietic pigment cells/ neural crest cells on the dorsum of the tail ${ }^{19}$ was unaffected (Fig. 4C, D; arrowheads). Control embryos injected with equal amounts of the negative control MO, pu.1 ex 5 mis (Fig. 4A, C), or pu.1 init mis (data not shown) did not affect staining with $l-p l$ or $c-f m s$. The costaining results combined with the pu.1 MO results demonstrated that there was loss of macrophage development in the absence of Pu.1.

While injection of either init or ex 5 pu. $1 \mathrm{MO}$ resulted in complete loss of l-pl and c-fms staining, expression of mpo and lys $C$ was unaffected in pu.1 MO-injected embryos (Fig. 4E$\mathrm{H})$ in three independent experiments. Only when a combination of the two MOs was injected at a maximal concentration of $0.375 \mathrm{mM}$ pu.1 init and $0.025 \mathrm{mM}$ pu.1 ex 5, was there loss or severe reduction of mpo expression in $92 \%$ of embryos $(n=38)$ and loss or severe reduction in lys $C$ expression in $67 \%$ of embryos $(n=45)$. Injection of higher doses of the combination was toxic, resulting in morphologic abnormalities and lethality (data not shown).

To determine whether the phenotypic changes seen with the MO injections were due to 


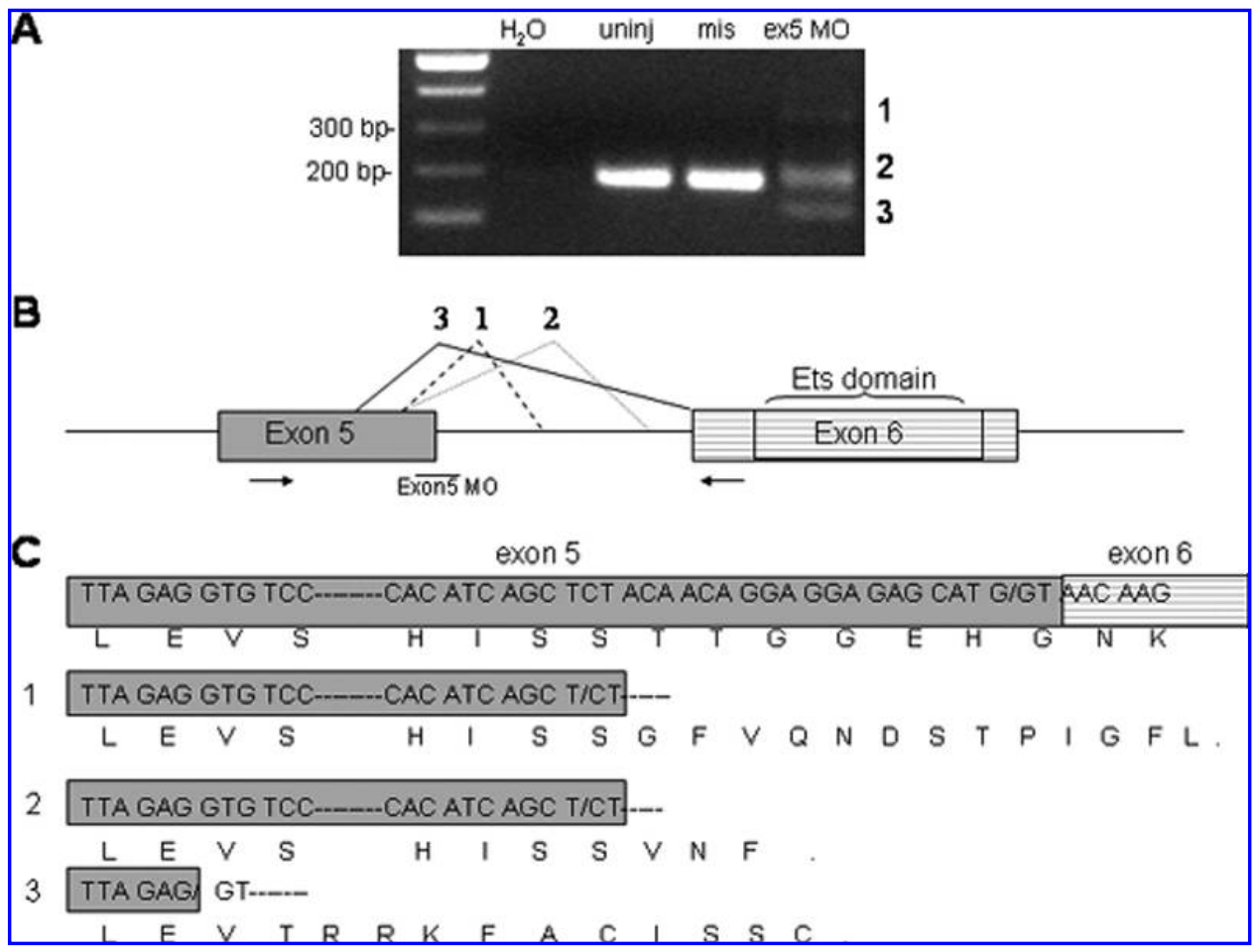

FIG. 3. RT-PCR with mRNA from pu.1 MO-injected embryos demonstrates aberrant splice products. (A) $2 \%$ agarose gel electrophoresis with water control $\left(\mathrm{H}_{2} \mathrm{O}\right)$, mRNA from uninjected embryos (uninj), pu.1 ex 5 mis embryos (mis), and pu.1 ex $5 \mathrm{MO}$ embryos (ex5 MO). Three aberrant splice products were amplified, designated 1, 2, and 3. PCR products were subcloned and sequenced. All products were different than the product amplified from uninjected and mis controls. (B) Schematic showing pu.1 exon 5 and exon 6. PCR primer locations are indicated by black arrow, and the region targeted by the exon $5 \mathrm{MO}$ is shown. The cryptic splice sites are drawn and labeled 1, 2, and 3 as labeled in the agarose gel in (A). (C) A region of DNA at the $3^{\prime}$ end of exon 5 and the $5^{\prime}$ end of exon 6 of pu. 1 is shown. The predicted translations for the splice products 1-3 all result in truncated products, with complete loss of the Ets domain encoded by exon 6 (shown with horizontal shading).

specific loss of Pu.1, rescue experiments with pu.1 mRNA were performed. mRNA synthesized in vitro from pu.1 cDNA will not be recognized by the ex $5 \mathrm{MO}$ since the splice donor site is absent in normally spliced mRNA. Injection of pu. 1 ex $5 \mathrm{MO}(0.1 \mathrm{mM})$ with pu. $1 \mathrm{mRNA}$ $(50 \mathrm{pg})$ resulted in rescue of $l-p l$ expression in $79 \%$ of embryos $(n=85)$ compared to $l-p l$ expression observed in only $13 \%$ of embryos injected with MO alone $(p<0.001)$ (Fig. 5A, B).

\section{c/ebp1 is required for lys $\mathrm{C}$ expression}

To evaluate the function of the transcription factor C/ebp1 in myeloid development, we used two translation initiation site MOs designated $c / e b p 1.1$ and $c / e b p 1.2$. Injection of either $\mathrm{MO}$ alone or the combination resulted in significant reduction or absence of lys $C$ expression by $24 \mathrm{hpf}$ in $85 \%$ of embryos $(n=79)$ compared to controls in which lys $C$ expression was positive in $100 \%$ of embryos $(n=32)$ (Fig. $6 \mathrm{E}, \mathrm{F})$. In contrast, $l-p l$ expression and mpo expression were unaffected (Fig. 6A-D). Further, expression of $c / e b p 1$ itself was significantly reduced or absent in $60-80 \%$ of embryos in three independent experiments (Fig. 6G, H), consistent with a model in which $\mathrm{C} / \mathrm{ebp} 1$ autoregulates its own expression.

$c / e b p 1$ mRNA (50 pg) lacking the $5^{\prime}$ untranslated region recognized by both $\mathrm{MOs}$ was coinjected with each $\mathrm{MO}$ to assess rescue. Rescue was incomplete, with lys $C$ expression in $68 \%$ of embryos coinjected with $\mathrm{MO}$ and mRNA $(n=25)$ compared to expression of lys $C$ in $15 \%$ of embryos injected with MO alone $(n=79)$ $(p<0.001)$. Injection of higher concentrations of mRNA resulted in gross morphologic abnormalities. It is likely that due to the highly conserved bZIP domain of $c / e b p 1$, which contains 


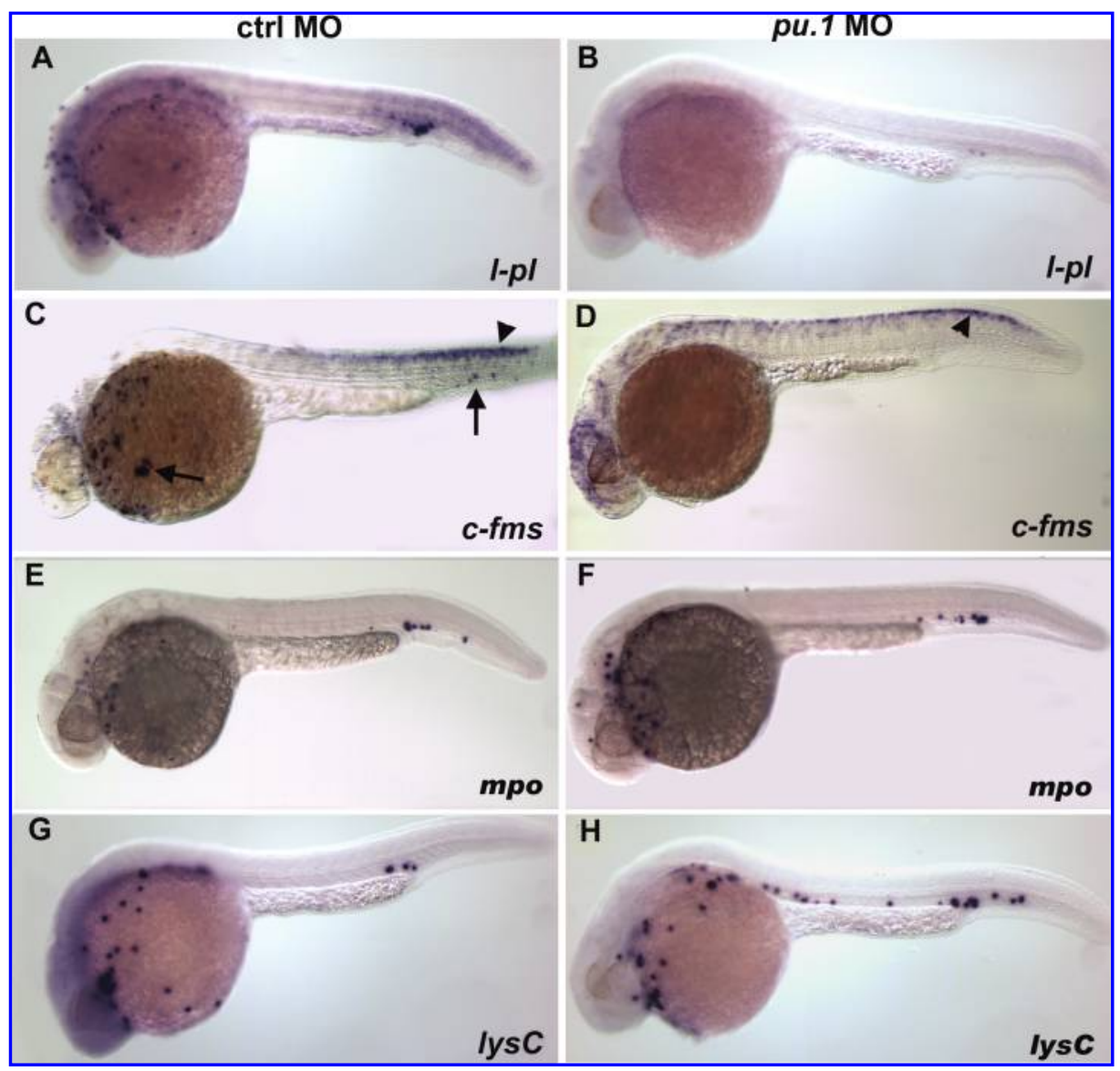

FIG. 4. Injection of ex 5 pu.1 MO results in loss of $l-p l$ and $c$-fms expression. All embryos are $28 \mathrm{hpf}$ and are shown in lateral view with head to the left. Embryo shown is representative of all embryos under specified condition. (A, C, E, and $\mathbf{G})$ pu.1 ex 5 mis MO-injected embryos (ctrl MO). (B, D, F, and $\mathbf{H})$ pu.1 ex 5 MO-injected embryos (pu.1 MO). In situ hybridizations were performed with the following markers: (A, B) $l-p l,(\mathbf{C}, \mathbf{D}) c-f m s,(\mathbf{E}, \mathbf{F}) m p o$, and $(\mathbf{G}, \mathbf{H}) l y s$ C. In (C), black arrows indicate myeloid-specific $c$-fms cell staining, and in (C) and (D), black arrowheads show neural crestderived pigment cell $c$-fms staining.

the dimerization and DNA binding regions, widespread ectopic expression of $c / e b p 1$ may activate many genes controlled by the other C/ebp family members. In support of this speculation, in previous work it was also not possible to inject gata1 mRNA without encoun- tering toxicity, also likely due to ectopic activation of many Gata-responsive genes. ${ }^{8}$

To confirm the results of the c/ebp $1 \mathrm{MO}$ knockdown, a $\gamma$-ray deletion mutant, c1054, which has a deletion in the distal portion of linkage group 24 (M.E. Halpern and A. Fritz,

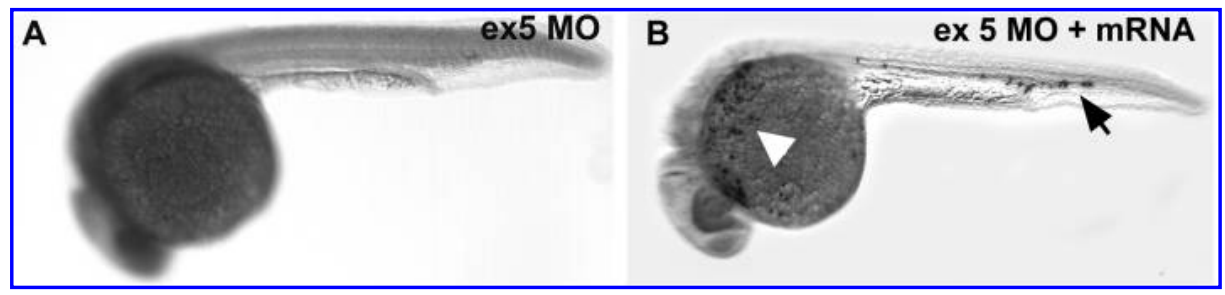

FIG. 5. Rescue of $l-p l$ expression by pu.1 mRNA. Lateral views at $24 \mathrm{hpf}$ are shown, with heads to the left. Embryos were injected with pu.1 ex $5 \mathrm{MO}$. Approximately $50 \mathrm{pg}$ negative control mRNA was injected in (A) and 50 pg of pu.1 mRNA was coinjected in (B). Rescue of $l-p l$ expression is shown. 


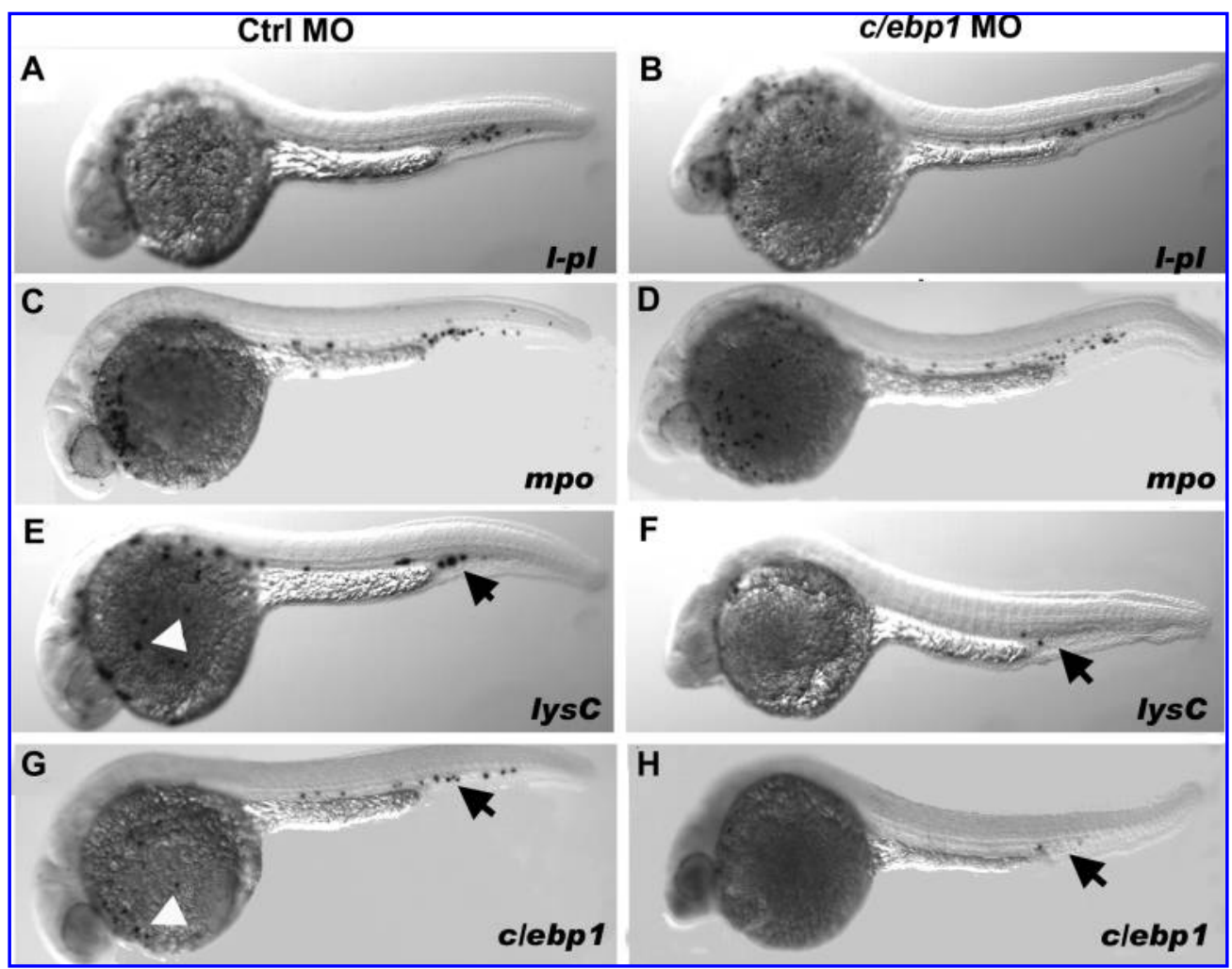

FIG. 6. Embryos injected with $c / e b p 1$ MOs demonstrate absence of $l y s C$ and $c / e b p 1$ expression. All embryos are shown in lateral view with head to the left. $(\mathbf{A}, \mathbf{C}, \mathbf{E}$, and G) $c / e b p 1$ mis $1 \mathrm{MO}$-injected embryos (Ctrl MO). (B, D, F, and H) $c / e b p 1$ MO-injected embryos. In situ hybridizations were performed with the following markers: (A, B) $l$ - $p l,(\mathbf{C}, \mathbf{D}) m p o,(\mathbf{E}, \mathbf{F})$ lys $C$, and $(\mathbf{G}, \mathbf{H}) c /$ ebp1. $(\mathbf{G}, \mathbf{I})$ Black arrows indicate expression of the indicated marker in the tail of control embryos $(\mathbf{E}, \mathbf{G})$ with minimal staining in $c / e b p 1 \mathrm{MO}-$ injected embryos $(\mathbf{F}, \mathbf{H})$. White arrowheads indicate staining in yolk sac.

unpublished data), where $c / e b p 1$ was previously mapped, ${ }^{17}$ was examined. We analyzed the deleted region in this mutant by amplification from c1054 genomic DNA with primers within $c / e b p 1$ and flanking markers. These data showed that the deleted region occurred distal to $64.5 \mathrm{cM}$ and spanned at least $71-72.1 \mathrm{cM}$, including deletion of $c / e b p 1$ (Fig. 7A, B). While multiple genes are likely included in the deleted region, the loss of $c / e b p 1$ provided an opportunity to confirm the MO results in homozygous c1054 mutant embryos.

The mutant embryos displayed normal expression of the macrophage marker $c$-fms $(n=11)$ (Fig. 7C, D) and the granulocyte marker mpo $(n=17)$ (Fig. 7E), but had little or no expression of lys $C(n=23)$ (Fig. 7F), confirming the findings in the $c / e b p 1 \mathrm{MO}$-injected embryos. Thus, in the absence of $\mathrm{C} / \mathrm{ebp} 1$ expression, embryos displayed normal $l-p l, c-f m s$, and mpo expression, allowing us to draw the conclusion that macrophages and granulocytes were present, but either did not express lys $C$ or lacked the granules containing lys $C$.

\section{Discussion}

The use of the zebrafish for dissection of developmental pathways has gained prominence over the past decade. While many mutants affecting erythroid development have been isolated, few mutants with myeloid defects have been identified. ${ }^{22}$ Identification of genes expressed in myeloid cells has now allowed the development of myeloid-specific mutagenesis screens, but a detailed understanding of the normal myelopoietic pathway is still lacking.

An issue of great importance to these analyses is the comparison of primitive myeloid development in the zebrafish to the myeloid processes that occur in higher vertebrates. In zebrafish, both granulocytes and macrophages develop between 21 somites (19.5 hpf) and 


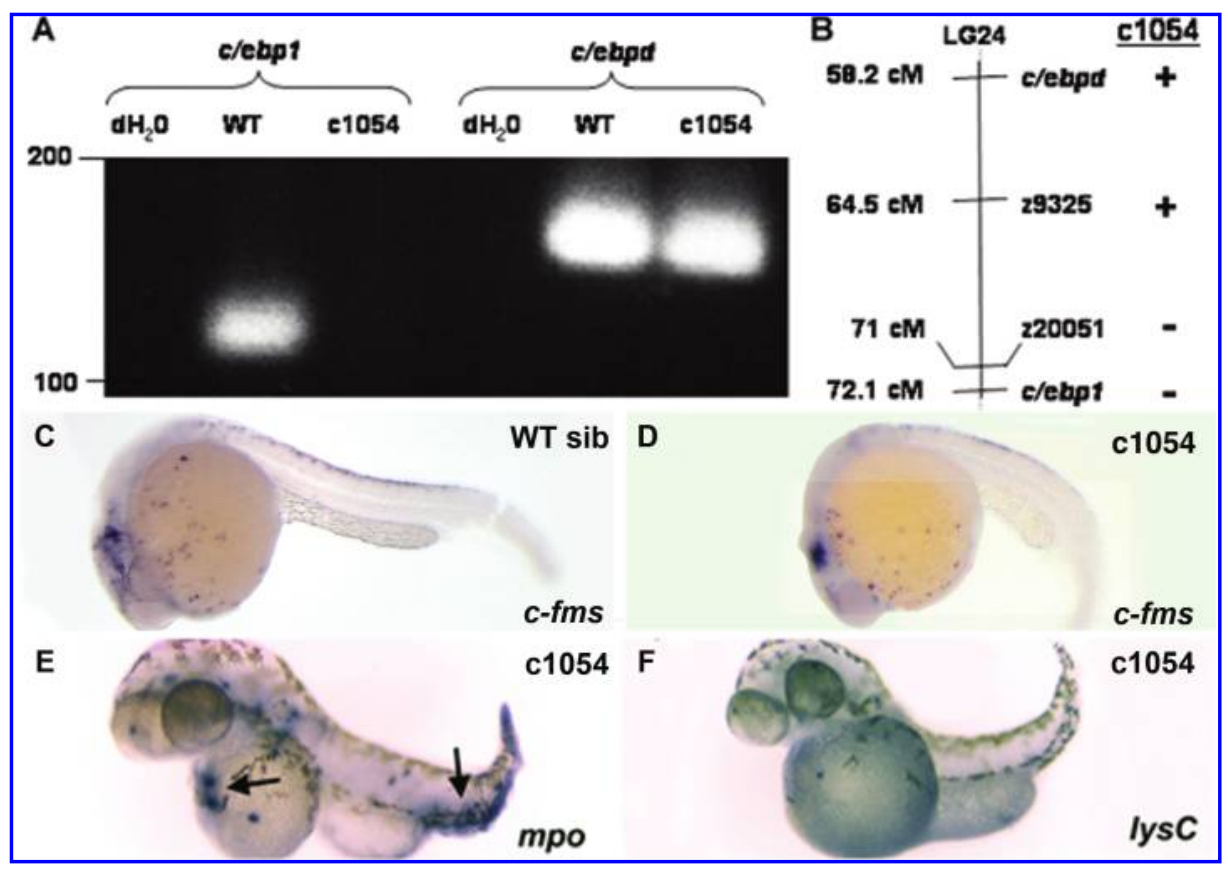

FIG. 7. Deletion mutant lacking $c / e b p 1$ has loss of $l y s$ C. (A) $2 \%$ agarose gel with products amplified from genomic DNA. c/ebp1 primers amplified a product from wild-type (WT) fish genomic DNA, but not from the c1054 deletion mutant. c/ebpd primers amplified product from both WT and c1054 deletion mutant. (B) Linkage group 24 map showing relative positions of $c / e b p 1$ and $c / e b p d$. To the right of the map are shown $(+)$ signs for DNA present in the c1054 mutant, including c/ebpd and z9325 (data not shown). The (-) signs indicate DNA that is absent in the deletion mutant, z20051 (data not shown), and c/ebp1. (C) Wild-type sibling (WT sib) at 24 hpf shows expression of $c-f m s$. (D) c1054 deletion homozygote at $24 \mathrm{hpf}$ is stunted in appearance, but shows normal staining with c-fms. (E, F) $48 \mathrm{hpf}$ c1054 deletion homozygotes are shown at $24 \mathrm{hpf}$ in lateral position. While they have deformities due to the deletion in linkage group 24, staining with (E) mpo is easily visible (shown by arrows), while there was no staining with (F) lys $C$.

3 days postfertilization (dpf), during the primitive wave of hematopoiesis., ${ }^{9,11}$ The great advantage of zebrafish is the ability to analyze these early developmental events. However, it is unclear how primitive myeloid development in the zebrafish compares to primitive macrophage and, more importantly, definitive myeloid development in higher vertebrates.

Our studies reveal that two distinct myeloid cell types can be distinguished in primitive myelopoiesis by whole-mount RNA in situ hybridization. The mpo/lys $C$ double-staining population labels the neutrophil/heterophil population. In mammals, lysozyme expression has been documented in both granulocytes and macrophages. However, an in vivo tagging study, using a Lysozyme promoter to drive green fluorescent protein (GFP) expression in mice, demonstrated that GFP was most highly expressed in granulocytes, with much lower expression in myelocyte progenitors and monocytic cells. ${ }^{23}$ Our findings are consistent with that work, showing that lysozyme is most highly expressed in granulocytes.

The $l-p l(+)$ population in zebrafish has been previously identified as a macrophage population based on correlation of video microscopy findings and $l-p l(+)$ cells by RNA in situ hybridization. ${ }^{10,20}$ In humans, L-PL is expressed in granulocytes, monocytes/macrophages, lymphocytes, and natural killer cells, and is frequently expressed in both hematopoietic and nonhematopoietic malignant cells. ${ }^{24}$ In contrast, $C-F M S$, encoding the macrophage colonystimulating factor receptor, is expressed specifically in macrophages. ${ }^{25}$ The coexpression of c-fms and l-pl, combined with previous work correlating live macrophage movements with in situ findings, ${ }^{10,20}$ demonstrates that the l-pl-expressing cells are likely the monocyte/ macrophage population.

We used two different MOs recognizing pu.1 to assess Pu.1 ablation in zebrafish. Either MO singly resulted in complete loss of $l-p l$ and $c-f m s$ 
expression, while mpo expression and lys $C$ expression were maintained, consistent with a specific loss of the macrophage lineage. When both MOs were injected together at the highest dosage possible without nonspecific toxicity, a significant loss of mpo and lys $C$ expression could be achieved. These data suggest that a differential threshold level of Pu.1 is needed for macrophage development and granulocyte development.

A number of studies in mammals have identified graded requirements of Pu.1 for development of hematopoietic lineages. In vivo, two independent Pu.1 null mouse models have been characterized. ${ }^{26,27}$ In both, development of monocyte/macrophages was deficient, while effects on neutrophil development were more varied. Additional work with both models showed that granulocyte commitment and early gene expression, including expression of Mpo, occurred in the absence of Pu.1. ${ }^{28-30}$ However, formation of terminally differentiated, functional neutrophils did not occur without Pu.1.

Studies in tissue culture have demonstrated that higher levels of Pu.1 are required for macrophage development than for granulocyte ${ }^{31}$ or B-cell development. ${ }^{32}$ Our work demonstrates a definite requirement for pu.1 in macrophage development, consistent with both mouse models and in vitro studies. Granulocytes were able to develop in the zebrafish with very low levels of Pu.1 in our studies.

Recent work using a single pu.1 initiation $\mathrm{MO}$ in zebrafish embryos demonstrated loss of both macrophage and granulocyte development with a single initiation site $\mathrm{MO}{ }^{33}$ It is possible that the differences between our findings and those of Rhodes et al..$^{33}$ may be due to greater Pu.1 knockdown using their MO. Interestingly, the translation initiation pu.1 MO used by Rhodes et al. ${ }^{33}$ does not target the first initiation ATG as did our init $\mathrm{MO}$, but rather the fifth in-frame ATG. It is possible that translation occurs from some of these more downstream in-frame ATGs, which might have resulted in continued protein expression with our translation initiation site MO.

Our analysis of the function of C/ebp1 demonstrated a role either in expression of lys $C$ or in myeloid development downstream of mpo, but upstream of lys C. c/ebp1 is a myeloidspecific $c / e b p$ without a mammalian ortholog. ${ }^{17}$
While orthologs for $C / E B P A$ and $C / E B P B$ have been identified in the zebrafish, a zebrafish ortholog for C/EBPE has not been identified in library screens ${ }^{16}$ or analysis of the available genetic databases (S.E. Lyons, unpublished results).

We have postulated that C/ebp1 may perform an analogous function with $\mathrm{C} / \mathrm{EBP}$. In humans and mice, $\mathrm{C} / \mathrm{EBP} \varepsilon$ is required for expression of secondary granule genes, as shown both by mouse gene ablation studies ${ }^{34-36}$ and by the naturally occurring human disorder due to mutations in $C / E B P \varepsilon$, neutrophil-specific granule deficiency. ${ }^{35,37,38}$ Myeloperoxidase is an enzyme of primary granules, while lysozyme is an enzyme found in both primary and secondary granules. ${ }^{39,40}$ Additional secondary granule proteins have not yet been identified in zebrafish. Our finding of mpo expression preceding lys $C$ expression in cells in the ICM suggests that these cells may be earlier myeloid progenitors. The isolated loss of lys $C$ expression after $\mathrm{C} / \mathrm{ebp} 1$ ablation is consistent with a role for $\mathrm{C} / \mathrm{ebp} 1$ in terminal myeloid differentiation. We can hypothesize that $\mathrm{C} / \mathrm{ebp} 1$ may regulate lys $C$ or may act upstream of secondary granule formation in granulocytes. The assessment of C/ebp1 function in zebrafish myeloid development suggests that it may play a functionally conserved role with mammalian C/EBP $\varepsilon^{41}$

Our data show that primitive myeloid development in zebrafish results in at least two myeloid lineages, distinguishable by multiple markers in RNA in situ hybridization. Using pu.1 and $c / e b p 1 \mathrm{MOs}$, we have begun to construct the developmental pathways of primitive myeloid development in the zebrafish (Fig. 8). The function of Pu.1 appears to be highly conserved from mammalian definitive myelopoiesis to zebrafish primitive myelopoiesis. The functions of $\mathrm{C} / \mathrm{ebp} 1$ may be conserved with mammalian $\mathrm{C} / \mathrm{EBP} \varepsilon$.

These studies demonstrate that zebrafish primitive myeloid development can be used as a model system with direct relevance to human myeloid lineage development. They also reveal the complexity of the myeloid pathway, and the need to tailor mutagenesis screens to identify myeloid mutants. The choice of the myeloidspecific marker used in a mutagenesis screen will determine the genes and specific pathways that will be identified. Identification and characterization of mutants with defects in myeloid 


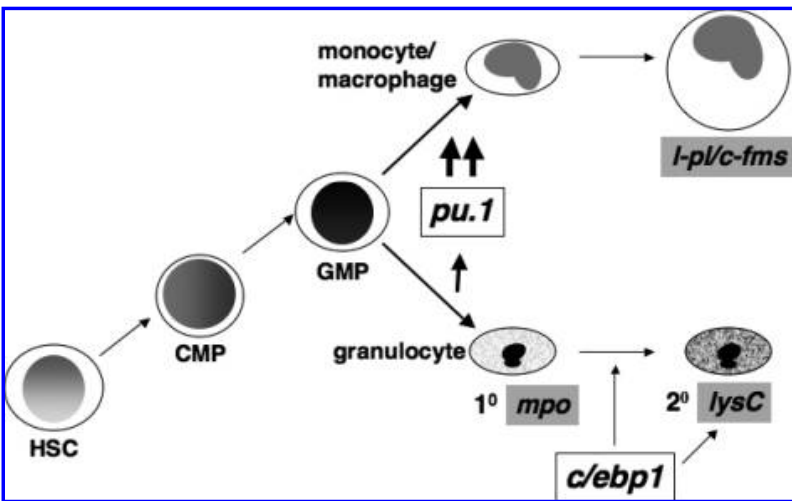

FIG. 8. Schematic of primitive myelopoiesis in zebrafish. A proposed developmental pathway for myelopoiesis in zebrafish embryos is shown with the transcription factors Pu.1 and C/ebp1 placed in the pathway. Higher levels of $\mathrm{Pu} .1$ are needed for macrophage development, while low levels are sufficient for granulocyte development, similar to the gradient required in mammalian hematopoiesis. $\mathrm{C} / \mathrm{ebp} 1$ is shown upstream of secondary granule formation or regulating lys $C$ expression in granulocyte development.

marker expression has the potential to identify genes encoding direct upstream regulators of myeloid proteins and effectors of lineagedetermination decisions. Future studies of zebrafish embryonic myelopoiesis will greatly enhance our understanding of the myeloid developmental pathway in vertebrates.

\section{Materials and Methods}

\section{Zebrafish maintenance and breeding}

Zebrafish were raised and maintained as described previously, ${ }^{42}$ with EK strain wild-type fish used for all studies. Embryos older than $24 \mathrm{hpf}$ were treated with $0.003 \%$ 1-phenyl-2thiourea (Sigma, St. Louis, MO) to inhibit pigment formation.

\section{Whole-mount RNA in situ hybridization}

Embryos were collected and whole-mount in situ hybridization was performed as previously described ${ }^{17}$ with minor modifications. Embryos older than $24 \mathrm{hpf}$ were treated with proteinase $\mathrm{K}$ treatment at $10 \mu \mathrm{g} / \mathrm{mL}$ for $5 \mathrm{~min}$, embryos at $2 \mathrm{dpf}$ with $20 \mu \mathrm{g} / \mathrm{mL}$ for $20 \mathrm{~min}$, and embryos at 3-4 dpf with $100 \mu \mathrm{g} / \mathrm{mL}$ for $20 \mathrm{~min}$. Hybridization with probes and the subsequent washes were all performed at $60^{\circ} \mathrm{C}$.

Antisense RNAs for in situ hybridization were prepared as previously reported for lys $C_{1}^{18}$ mpo, ${ }^{9}$ l-pl, ${ }^{10}$ c-fms, ${ }^{19}$ gata $1,{ }^{13}$ and c/ebp $1 .{ }^{17}$ Probes were synthesized with digoxigenin (DIG)labeled uridine triphosphates (UTPs) according to the manufacturer's instructions (Roche, Basel, Switzerland) or with fluorescein (FLUOR)labeled UTPs in the case of double staining.

For two-color staining, in situ hybridization was performed as previously described. ${ }^{17}$ For fluorescence two-color staining, embryos were hybridized with both DIG-labeled and FLUORlabeled probes followed by posthybridization incubation with anti-DIG-alkaline phosphatase and anti-FLUOR horseradish peroxidase (HRP) antibodies. ${ }^{43,44}$ Color/fluorescent staining was performed with Fast Red (Roche), which formed a color precipitate that is also visible under fluorescence using a rhodamine filter. The HRPconjugated antibody results in fluorescence detectable with a fluorescein isothiocyanate (FITC) filter using the tyramide signal amplification (TSA)-biotin system (Perkin-Elmer, Waltham, MA). Embryos were visualized on a Leica MZFLIII dissecting microscope and photographed with a Coolsnap digital camera.

\section{MO microinjection}

Embryos used for microinjection were obtained from breeding wild-type EK strain zebrafish. The following MOs were created by GENE TOOLS, LLC (Philomath, OR; www .Gene-Tools.com). Lower-case letters designate mismatches, and underscore marks initiation ATG.

pu.1 init (initiation site MO): 5'-CCTCCATTCTGTAC GGATGCAGCAT-3'

pu.1 init mis (initiation site mismatch): $5^{\prime}$-CgTCgATTC TcTACGGATGgAGgAT-3'

pu.1 ex 5 (exon 5 splice MO): 5'-GGTCTTTCTCCTTAC CATGCTCTCC- $3^{\prime}$

pu.1 ex 5 mis (exon 5 mismatch MO): 5'-GGTgTTTgTC CTTAgCATcCTgTCC-3'

c/ebp1 1 (initiation MO 1): 5'-CACCGACATGGCTGT GTGTGGAGCT- ${ }^{\prime}$

c/ebp1 2 (initiation MO 2): 5'-TGCTGAACTCTACTCG ATCTCGTCC-3'

c/ebp1 mis 1 (init 1 mismatch): 5'-CAgCGAgATGggTG TGTGTcGAcCT-3'

c/ebp1 mis 2 (init 2 mismatch): 5'-TcCTcAACTCTAgTC GATCTgGTgC-3'

MOs were injected in volumes of approximately $2 \mathrm{~nL}$ at concentrations ranging from 0.05 to $1.0 \mathrm{mM}$ (total quantities injected were 
approximately $1-20 \mathrm{ng}$ ). For each $\mathrm{MO}$ or $\mathrm{MO}$ mix, injection concentrations were titrated to determine the maximal injectable dose with minimal nonspecific morphologic abnormalities. MO concentrations injected were as follows: pu.1 init, $0.75 \mathrm{mM}$; pu.1 ex 5, $0.1 \mathrm{mM}$; pu.1 init/ex 5, $0.375 \mathrm{mM} / 0.025 \mathrm{mM}$; c/ebp1 init $1 / 2$, $0.25 \mathrm{mM}$ each. The same concentrations of mismatch were used for each corresponding MO. Microinjections were performed according to published protocols ${ }^{42}$ using a pneumatic picopump (World Precision Instruments, Sarasota, FL). All results were obtained in at least three independent experiments with at least 20 embryos/MO condition.

\section{Analysis of mRNA splice products}

After injection of embryos with pu.1 ex 5 or ex $5 \mathrm{mis}$ MOs, embryos were collected into Trizol (Invitrogen, Carlsbad, CA) at the 21 somite stage. RNA was isolated according to manufacturer's instructions. mRNA was used for a reverse transcriptase reaction using random oligonucleotides as primers followed by PCR using the following primers targeting the pu. 1 cDNA between exon 5 and exon 6 :

$$
\begin{aligned}
& \text { pu.1 exon } 5 \text { forward: 5'-GACATCGGTGTGTTACCCT } \\
& \text { C-3' } \\
& \text { pu.1 exon } 6 \text { reverse: 5'-AGCAGGAACTGATACAAGC } \\
& \text { G-3'. }
\end{aligned}
$$

For analysis of the deleted region of the c1054 $\gamma$-ray mutant, genomic DNA from a wild-type control and c1054 were amplified with $\mathrm{z}$ marker primers and primers for $c / e b p 1$ and c/ebpd described previously. ${ }^{16,17}$

\section{$m R N A$ rescue}

pu.1 and c/ebp1 cDNAs were amplified and subcloned into pCS $2+{ }^{45}$ digested with BamHI and EcoRI. For $c / e b p 1$, the forward primer begins amplification at the ATG, removing the $5^{\prime}$ untranslated sequence recognized by the $\mathrm{c} / \mathrm{ebp}$ MOs. The forward primers have a BamHI site underlined, and the reverse primers have an EcoRI site underlined:

c/ebp1 forward: 5'-CGGGATCCGGATGTCAGTCTCT GACAACATC-3'

c/ebp1 reverse: 5'-GCGAATTCTCAGTGCTCCTCAGA TGTG-3' pu.1 forward: 5'-CGGGATCCGGATGCTGCATCCGTA CAG-3'

pu.1 reverse: 5'-GCGAATTCTTACATGTAATGCTTTC TG-3'

Capped mRNA was synthesized using an mMessage RNA synthesis kit (Ambion, Foster City, CA) as previously described. ${ }^{46}$

Embryos were injected at the one-cell stage with appropriate MOs and 50-100 pg of mRNA. Rescue was evaluated by in situ hybridization for rescue of hematopoietic marker expression. Embryos injected with 50-100pg GFP mRNA were used as negative control embryos.

\section{Acknowledgments}

We greatly appreciate the gift of deletion mutant c1054 genomic DNA and embryos from Andreas Fritz and Marnie Halpern. We thank Dr. Doug Engel for critical reading of the manuscript.

\section{References}

1. Begley CG, Aplan PD, Davey MP, Nakahara K, Tchorz K, Kurtzberg J, et al. Chromosomal translocation in a human leukemic stem-cell line disrupts the $\mathrm{T}$-cell antigen receptor delta-chain diversity region and results in a previously unreported fusion transcript. Proc Natl Acad Sci USA 1989;86:2031-2035.

2. Mueller BU, Pabst T, Osato M, Asou N, Johansen LM, Minden MD, et al. Heterozygous PU.1 mutations are associated with acute myeloid leukemia. Blood 2002; 100:998-1007.

3. Pabst T, Mueller BU, Zhang P, Radomska HS, Narravula $S$, Schnittger $S$, et al. Dominant-negative mutations of CEBPA, encoding CCAAT/enhancer binding protein-alpha (C/EBPalpha), in acute myeloid leukemia. Nat Genet 2001;27:263-270.

4. Shimizu R, Kuroha T, Ohneda O, Pan X, Ohneda K, Takahashi $\mathrm{S}$, et al. Leukemogenesis caused by incapacitated GATA-1 function. Mol Cell Biol 2004;24: 10814-10825.

5. Crispino JD. GATA1 in normal and malignant hematopoiesis. Semin Cell Dev Biol 2005;16:137-147.

6. Shafizadeh E, Paw BH. Zebrafish as a model of human hematologic disorders. Curr Opin Hematol 2004;11: 255-261.

7. Brownlie A, Zon L. The zebrafish as a model system for the study of hematopoiesis. Zebrafish mutants point the way to novel genes involved in the generation of vertebrate blood cells. Bioscience 1999;49:382392.

8. Lyons SE, Lawson ND, Lei L, Bennett PE, Weinstein BM, Liu PP. A nonsense mutation in zebrafish gata1 causes the bloodless phenotype in vlad tepes. Proc Natl Acad Sci USA 2002;99:5454-5459. 
9. Bennett CM, Kanki JP, Rhodes J, Liu TX, Paw BH, Kieran MW, et al. Myelopoiesis in the zebrafish, Danio rerio. Blood 2001;98:643-651.

10. Herbomel P, Thisse B, Thisse C. Ontogeny and behaviour of early macrophages in the zebrafish embryo. Development 1999;126:3735-3745.

11. Lieschke GJ, Oates AC, Crowhurst MO, Ward AC, Layton JE. Morphologic and functional characterization of granulocytes and macrophages in embryonic and adult zebrafish. Blood 2001;98:3087-3096.

12. Bahary N, Zon LI. Use of the zebrafish (Danio rerio) to define hematopoiesis. Stem Cells 1998;16:89-98.

13. Detrich HW, 3rd, Kieran MW, Chan FY, Barone LM, Yee K, Rundstadler JA, et al. Intraembryonic hematopoietic cell migration during vertebrate development. Proc Natl Acad Sci USA 1995;92:10713-10717.

14. Fisher RC, Scott EW. Role of PU.1 in hematopoiesis. Stem Cells 1998;16:25-37.

15. Simon MC. PU.1 and hematopoiesis: lessons learned from gene targeting experiments. Semin Immunol 1998;10:111-118.

16. Lyons SE, Shue BC, Lei L, Oates AC, Zon LI, Liu PP. Molecular cloning, genetic mapping, and expression analysis of four zebrafish c/ebp genes. Gene 2001;281: 43-51.

17. Lyons SE, Shue BC, Oates AC, Zon LI, Liu PP. A novel myeloid-restricted zebrafish CCAAT/enhancerbinding protein with a potent transcriptional activation domain. Blood 2001;97:2611-2617.

18. Liu F, Wen Z. Cloning and expression pattern of the lysozyme C gene in zebrafish. Mech Dev 2002;113: 69-72.

19. Parichy D, Ransom D, Paw B, Zon L, Johnson S. An orthologue of the kit-related gene $\mathrm{fms}$ is required for development of neural crest-derived xanthophores and a subpopulation of adult melanocytes in the zebrafish, Danio rerio. Development 2000;127:3031-3044.

20. Herbomel P, Thisse B, Thisse C. Zebrafish early macrophages colonize cephalic mesenchyme and developing brain, retina, and epidermis through a M-CSF receptor-dependent invasive process. Dev Biol 2001; 238:274-288.

21. Tagoh H, Himes R, Clarke D, Leenen PJ, Riggs AD, Hume $\mathrm{D}$, et al. Transcription factor complex formation and chromatin fine structure alterations at the murine c-fms (CSF-1 receptor) locus during maturation of myeloid precursor cells. Genes Dev 2002;16:1721-1737.

22. Hogan BM, Layton JE, Pyati UJ, Nutt SL, Hayman JW, Varma $S$, et al. Specification of the primitive myeloid precursor pool requires signaling through Alk8 in zebrafish. Curr Biol 2006;16:506-511.

23. Faust N, Varas F, Kelly LM, Heck S, Graf T. Insertion of enhanced green fluorescent protein into the lysozyme gene creates mice with green fluorescent granulocytes and macrophages. Blood 2000;96:719-726.

24. Lin CS, Park T, Chen ZP, Leavitt J. Human plastin genes. Comparative gene structure, chromosome location, and differential expression in normal and neoplastic cells. J Biol Chem 1993;268:2781-2792.
25. Woolford J, Rothwell V, Rohrschneider L. Characterization of the human c-fms gene product and its expression in cells of the monocyte-macrophage lineage. Mol Cell Biol 1985;5:3458-3466.

26. Scott EW, Simon MC, Anastasi J, Singh H. Requirement of transcription factor PU.1 in the development of multiple hematopoietic lineages. Science 1994;265: 1573-1577.

27. McKercher SR, Torbett BE, Anderson KL, Henkel GW, Vestal DJ, Baribault H, et al. Targeted disruption of the PU.1 gene results in multiple hematopoietic abnormalities. EMBO J 1996;15:5647-5658.

28. Anderson KL, Smith KA, Conners K, McKercher SR, Maki RA, Torbett BE. Myeloid development is selectively disrupted in PU.1 null mice. Blood 1998;91: 3702-3710.

29. Anderson KL, Smith KA, Pio F, Torbett BE, Maki RA. Neutrophils deficient in PU.1 do not terminally differentiate or become functionally competent. Blood 1998;92:1576-1585.

30. Olson MC, Scott EW, Hack AA, Su GH, Tenen DG, Singh H, et al. PU.1 is not essential for early myeloid gene expression but is required for terminal myeloid differentiation. Immunity 1995;3:703-714.

31. Dahl R, Walsh JC, Lancki D, Laslo P, Iyer SR, Singh H, et al. Regulation of macrophage and neutrophil cell fates by the PU.1:C/EBPalpha ratio and granulocyte colony-stimulating factor. Nat Immunol 2003;4:10291036.

32. DeKoter RP, Singh H. Regulation of B lymphocyte and macrophage development by graded expression of PU.1. Science 2000;288:1439-1441.

33. Rhodes J, Hagen A, Hsu K, Deng M, Liu TX, Look AT, et al. Interplay of pu.1 and gata1 determines myeloerythroid progenitor cell fate in zebrafish. Dev Cell 2005;8:97-108.

34. Gombart AF, Kwok SH, Anderson KL, Yamaguchi Y, Torbett BE, Koeffler HP. Regulation of neutrophil and eosinophil secondary granule gene expression by transcription factors C/EBP epsilon and PU.1. Blood 2003;101:3265-3273.

35. Gombart AF, Koeffler HP. Neutrophil specific granule deficiency and mutations in the gene encoding transcription factor C/EBP(epsilon). Curr Opin Hematol 2002;9:36-42.

36. Yamanaka R, Barlow C, Lekstrom-Himes J, Castilla LH, Liu PP, Eckhaus M, et al. Impaired granulopoiesis, myelodysplasia, and early lethality in CCAAT/ enhancer binding protein epsilon-deficient mice. Proc Natl Acad Sci USA 1997;94:13187-13192.

37. Lekstrom-Himes JA, Dorman SE, Kopar P, Holland SM, Gallin JI. Neutrophil-specific granule deficiency results from a novel mutation with loss of function of the transcription factor CCAAT/enhancer binding protein epsilon. J Exp Med 1999;189:1847-1852.

38. Gombart AF, Shiohara M, Kwok SH, Agematsu K, Komiyama A, Koeffler HP. Neutrophil-specific granule deficiency: homozygous recessive inheritance of a frameshift mutation in the gene encoding transcription 
factor CCAAT/enhancer binding protein-epsilon. Blood 2001;97:2561-2567.

39. Cramer EM, Breton-Gorius J. Ultrastructural localization of lysozyme in human neutrophils by immunogold. J Leukoc Biol 1987;41:242-247.

40. Shaft D, Shtalrid M, Berebi A, Catovsky D, Resnitzky P. Ultrastructural characteristics and lysozyme content in hypergranular and variant type of acute promyelocytic leukaemia. Br J Haematol 1998;103:729-739.

41. Lekstrom-Himes JA. The role of C/EBP(epsilon) in the terminal stages of granulocyte differentiation. Stem Cells 2001;19:125-133.

42. Westerfield M. The Zebrafish Book: A Guide for the Laboratory Use of Zebrafish (Danio rerio), 3rd ed. University of Oregon Press, Eugene, Oregon, 1995.

43. Raymond PA, Barthel LK. A moving wave patterns the cone photoreceptor mosaic array in the zebrafish retina. Int J Dev Biol 2004;48:935-945.

44. Barthel LK, Raymond PA. In situ hybridization studies of retinal neurons. In: K. Palczewsk (ed). Methods in
Enzymology-Vertebrate Phototransduction and the Visual Cycle. Academic Press, Orlando, FL, 2000, pp. 579590.

45. Turner DL, Weintraub H. Expression of achaete-scute homolog 3 in Xenopus embryos converts ectodermal cells to a neural fate. Genes Dev 1994;8:1434-1447.

46. Juarez MA, Su F, Chun S, Kiel MJ, Lyons SE. Distinct roles for SCL in erythroid specification and maturation in zebrafish. J Biol Chem 2005;280:4163641644.

Address reprint requests to: Susan E. Lyons, M.D., Ph.D. 2215 Fuller Road Room B740 Ann Arbor, MI 48105

E-mail: suslyons@umich.edu 

This article has been cited by:

1. C. Liongue, C. J. Hall, B. A. O'Connell, P. Crosier, A. C. Ward. 2009. Zebrafish granulocyte colony-stimulating factor receptor signaling promotes myelopoiesis and myeloid cell migration. Blood 113:11, 2535-2546. [CrossRef]

2. Kevin B. Walters, M. Ernest Dodd, Jonathan R. Mathias, Andrea J. Gallagher, David A. Bennin, Jennifer Rhodes, John P. Kanki, A. Thomas Look, Yevgenya Grinblat, Anna Huttenlocher. 2009. Muscle degeneration and leukocyte infiltration caused by mutation of zebrafish fad24. Developmental Dynamics 238:1, 86-99. [CrossRef]

3. 2007. Recent Papers on Zebrafish and Other Aquarium Fish ModelsRecent Papers on Zebrafish and Other Aquarium Fish Models. Zebrafish 4:4, 297-308. [Citation] [PDF] [PDF Plus] 